\title{
UNDERSTANDING THE EVOLUTION OF CLOSE BINARY SYSTEMS WITH RADIO PULSARS
}

\author{
O. G. Benvenuto ${ }^{1,2}$, M. A. De Vito ${ }^{2}$, and J. E. Horvath ${ }^{3}$ \\ ${ }^{1}$ Facultad de Ciencias Astronómicas y Geofísicas, Universidad Nacional de La Plata, 1900 La Plata, Buenos Aires, Argentina; \\ obenvenu@fcaglp.unlp.edu.ar, adevito@fcaglp.unlp.edu.ar \\ 2 Instituto de Astrofísica de La Plata (IALP), CCT-CONICET-UNLP. Paseo del Bosque S/N (B1900FWA), La Plata, Argentina \\ ${ }^{3}$ Instituto de Astronomia, Geofísica e Ciências Atmosféricas, Universidade de São Paulo R. do Matão 1226 (05508-090), \\ Cidade Universitária, São Paulo SP, Brazil; foton@astro.iag.usp.br \\ Received 2013 November 18; accepted 2014 February 27; published 2014 April 14
}

\begin{abstract}
We calculate the evolution of close binary systems (CBSs) formed by a neutron star (behaving as a radio pulsar) and a normal donor star, which evolve either to a helium white dwarf (HeWD) or to ultra-short orbital period systems. We consider X-ray irradiation feedback and evaporation due to radio pulsar irradiation. We show that irradiation feedback leads to cyclic mass transfer episodes, allowing CBSs to be observed in between episodes as binary radio pulsars under conditions in which standard, non-irradiated models predict the occurrence of a low-mass X-ray binary. This behavior accounts for the existence of a family of eclipsing binary systems known as redbacks. We predict that redback companions should almost fill their Roche lobe, as observed in PSR J1723-2837. This state is also possible for systems evolving with larger orbital periods. Therefore, binary radio pulsars with companion star masses usually interpreted as larger than expected to produce HeWDs may also result in such quasi-Roche lobe overflow states, rather than hosting a carbon-oxygen WD. We found that CBSs with initial orbital periods of $P_{i}<1$ day evolve into redbacks. Some of them produce low-mass HeWDs, and a subgroup with shorter $P_{i}$ becomes black widows (BWs). Thus, BWs descend from redbacks, although not all redbacks evolve into BWs. There is mounting observational evidence favoring BW pulsars to be very massive ( $\left.\gtrsim 2 M_{\odot}\right)$. As they should be redback descendants, redback pulsars should also be very massive, since most of the mass is transferred before this stage.
\end{abstract}

Key words: binaries: close - pulsars: general - stars: evolution - stars: neutron

Online-only material: color figure

\section{INTRODUCTION}

Close binary systems (CBSs) formed by a neutron star (NS) orbiting together with a normal donor star experience a mass transfer episode wherein the donor star undergoes Roche lobe overflow (RLOF). From here, the stars are forced to exchange mass. A fraction $\beta \leqslant 1$ of the transferred matter, carrying a substantial amount of angular momentum, is accreted by the NS, which is recycled and may reach spin periods in the millisecond range. In the standard treatment, the initial RLOF is a long-lived episode; the donor star transfers a large fraction of its mass and the orbit is considerably affected. During RLOF episodes, a CBS can be detected as a low-mass X-ray binary (LMXB). For CBSs with initial orbital periods $P_{i}>1$ day, after the end of the RLOF, the pair may be observed as a radio pulsar with a binary companion, and almost no further orbital evolution is expected. Within this general picture, if we restrict ourselves to the case of stars that do not ignite helium, binary evolution predicts a tight donor-mass-orbital period ${ }^{4} P\left(M_{2}\right)$ relation (Tauris \& Savonije 1999; Podsiadlowski et al. 2002; De Vito \& Benvenuto 2012). Thus, for CBSs with $P_{i}>1$ day, standard calculations that lead to the formation of helium white dwarfs (HeWDs) predict the occurrence of companions to radio pulsars only in the neighborhood of the $P\left(M_{2}\right)$ relation. Currently, companions to radio pulsars more massive than predicted by the $P\left(M_{2}\right)$ relation are considered $\mathrm{CO}$ or HeCO WDs (Tauris et al. 2000; Podsiadlowski et al. 2002).

Two separate classes of interacting binary systems with radio pulsars have been identified; they are called redbacks and black

\footnotetext{
4 We denote the corresponding quantities of the NS and donor star with subscripts 1 and 2, respectively.
}

widows (BWs). Redbacks are eclipsing binary systems with 0.1 day $<P<1$ day and $0.2 M_{\odot}<M_{2}<0.4 M_{\odot}$. BWs have $P$ in the same range of values, but much lower companion masses $\left(M_{2}<0.05 M_{\odot}\right)$.

Standard CBS models do not take into account either evaporation of the donor star by radio pulsar irradiation (Stevens et al. 1992), or X-ray irradiation feedback (Büning \& Ritter 2004). During an RLOF, matter falling onto the NS produces X-ray radiation that illuminates the donor star, giving rise to the irradiation feedback phenomenon. If the irradiated star has an outer convective zone, its structure is considerably affected. Vaz \& Nordlund (1985) studied irradiated grey atmospheres, finding that the entropy at deep convective layers must be the same for the irradiated and non-irradiated portions of the star. To fulfill this condition, the irradiated surface is partially inhibited from releasing energy emerging from its deep interior, i.e., the effective surface becomes smaller than $4 \pi R_{2}^{2}\left(R_{2}\right.$ is the radius of the donor star). Irradiation makes the evolution depart from that predicted by the standard theory. After the onset of the RLOF, the donor star relaxes to the established conditions on a thermal (Kelvin-Helmholtz) timescale, $\tau_{\mathrm{KH}}=\mathrm{GM}_{2}^{2} /\left(\mathrm{R}_{2} \mathrm{~L}_{2}\right)(\mathrm{G}$ is the gravitational constant and $\mathrm{L}_{2}$ is the luminosity of the donor star). In some cases, the structure is unable to sustain the RLOF and becomes detached. Subsequent nuclear evolution may lead the donor star to experience RLOF again, undergoing a quasicyclic behavior (Büning \& Ritter 2004). Thus, irradiation feedback may lead to the occurrence of a large number of short-lived RLOFs instead of a long one. In between episodes, the system may reveal itself as a radio pulsar with a binary companion. Notably, the evolution of several quantities is only mildly dependent on the irradiation feedback (e.g., the orbital period). 
On the other hand, evaporation of the donor star due to radio pulsar irradiation affects the later evolution of CBSs, which eventually reaches very short orbital periods. This effect makes the donor star reach very low masses and makes these systems achieve orbital periods large enough to be compatible with observations (Benvenuto et al. 2012, 2013).

It is the aim of this work to explore the consequences of evaporation and X-ray irradiation feedback in the evolution of these CBSs. As stated above, CBSs undergo cyclic RLOFs. This allows us to interpret that companions to radio pulsars that are more massive than predicted by the $P\left(M_{2}\right)$ relation may also be progenitors of HeWDs or ultra-short orbital period systems that are observed between two consecutive RLOFs. This scenario provides a natural explanation for the occurrence of redback pulsars, as well as their evolutionary link with BWs.

The remainder of the paper is as follows. In Section 2, we describe our numerical evolutionary code. In Section 3, we present our models, while in Section 4, we interpret and discuss these results and give some concluding remarks.

\section{NUMERICAL TREATMENT}

We compute CBS evolution employing our code (Benvenuto $\&$ De Vito 2003), which has been updated to include irradiation feedback and evaporation. During an RLOF episode the code solves the structure of the donor star simultaneously with the instantaneous mass transfer rate $\dot{M}$, the evolution of both masses, and the orbital semi-axis in a fully implicit way. This strategy has the very desirable property of numerical stability (Büning $\&$ Ritter 2006) that allows for the computation of mass transfer cycles (Büning \& Ritter 2004; Benvenuto et al. 2012) presented in this Letter. When the pair becomes detached, the code follows the standard Henyey technique.

Let us remark on some key assumptions in our calculations. We assumed that the NS accretes a $\beta$ fraction of the transferred matter up to the Eddington critical rate $\dot{M}_{\text {Edd }} \approx 2 \times 10^{-8} M_{\odot} \mathrm{yr}^{-1}$, while very low accretion rates $\left(\lesssim 1.3 \times 10^{-11} M_{\odot} \mathrm{yr}^{-1}\right)$ are prevented by the propeller mechanism (Romanova et al. 2008). Angular momentum sinks due to gravitational radiation, magnetic braking, and mass loss from the system were treated as in Benvenuto \& De Vito (2003).

Irradiation feedback was included following Hameury \& Ritter (1997). The usual relation among luminosity, radius, and effective temperature $L=4 \pi R_{2}^{2} \sigma T_{\mathrm{eff}}^{4}$ for the case of an irradiated photosphere is replaced by

$$
L=R_{2}^{2} \sigma T_{\mathrm{eff}, 0}^{4} \int_{0}^{2 \pi} \int_{0}^{\pi} G(x(\theta, \phi)) \sin \theta d \theta d \phi,
$$

where $\sigma$ is the Stefan-Boltzmann constant, $T_{\mathrm{eff}, 0}$ is the effective temperature of the non-illuminated part of the star, $G(x)=\left(T_{\mathrm{eff}}(x) / T_{\mathrm{eff}, 0}\right)^{4}-x$, and $x=F_{\text {irr }} /\left(\sigma T_{\mathrm{eff}, 0}^{4}\right)$, where $F_{\text {irr }}$ is the incident irradiating flux. We find $T_{\text {eff }}(x)$ by assuming that at the deep layers, the perturbations due to irradiation should vanish (Vaz \& Nordlund 1985). We divided the irradiated part of the stellar surface into several sectors and solved for $T_{\text {eff }}(x)$ in each of them, and integrated over the illuminated zone. We have ignored any heat transfer between irradiated and non-irradiated zones. This imposes a limitation to the strength of the irradiation that our code can handle: $x \lesssim 10^{4}$ (Büning \& Ritter 2004). All our models are well below this limit.

We assume that the NS acts as a point source, releasing an accretion luminosity $L_{\text {acc }}=G M_{1} \dot{M}_{1} / R_{1}\left(M_{1}, R_{1}\right.$, and $\dot{M}_{1}$ are the mass, radius, and accretion rate of the NS, respectively).
Assuming isotropy, the energy flux incident onto the donor star is $F_{\text {irr }}=\alpha_{\text {irr }} L_{\text {acc }} /\left(4 \pi a^{2}\right)$, where $\alpha_{\text {irr }} \leqslant 1$ is the fraction of the incident flux that effectively irradiates the donor star and $a$ is the orbital radius.

Evaporation of the donor star due to radio pulsar irradiation is included by assuming a mass loss rate of (Stevens et al. 1992)

$$
\dot{M}_{2, \text { evap }}=-\frac{\alpha_{\text {evap }}}{2 v_{2, \text { esc }}^{2}} L_{\mathrm{PSR}}\left(\frac{R_{2}}{\mathrm{a}}\right)^{2}
$$

where $L_{\mathrm{PSR}}$ is the radio pulsar's spindown luminosity, $v_{2 \text {,esc }}$ is the escape velocity at the donor star surface, and $\alpha_{\text {evap }} \leqslant 1$ is an efficiency factor. $L_{\mathrm{PSR}}$ is a function of the spin and moment of inertia evolution of the NS, which depends upon the free parameter $\beta$. These quantities, as well as $\alpha_{\text {evap, are }}$ uncertain. So, as a first approximation we consider the product $\alpha_{\text {evap }} L_{\text {PSR }}$ to be time independent and adopt a range of plausible values.

Previous calculations (Benvenuto et al. 2012) indicate that evaporation is relevant for CBSs with very short orbital periods only, especially after these CBSs reach the minimum value of their periods. Because of this reason, we considered that evaporation begins to operate when the minimum orbital period is reached.

"Radio ejection" may be relevant for these CBSs. It has been proposed (Ruderman et al. 1989; Burderi et al. 2001) that the pressure due to radio pulsar irradiation may inhibit material lost by the donor star from being accreted by the NS. If "radio ejection" operates, it precludes irradiation feedback from occurring. In addition, we have neglected the presence of an accretion disk surrounding the NS. van Haaften et al. (2012) studied the evolution of ultra-compact X-ray binary systems, considering an accretion disk, allowing for the occurrence of short-timescale disk instabilities, and modulating the long-term evolution computed here. Including "radio ejection" and/or accretion disks is beyond the scope of this Letter.

\section{RESULTS}

We computed CBSs that initially have donor masses from $1.00 M_{\odot}$ to $3.50 M_{\odot}$ with steps of $0.25 M_{\odot}$, a "canonical" NS $\left(M_{1}=1.4 M_{\odot}\right)$, and $P_{i}$ of $0.50,0.75,1.00,1.50,3.00$, 6.00, and 12 days. We set $\beta=0.5$ for all calculations. At least for models without irradiation, donor evolution is almost independent of $\beta$. If $\beta<0.5$, irradiation will be weaker and NSs lighter. Pulsed mass transfer is found for $\alpha_{\text {irr }}>0$. Thus, lowering $\beta$ should not change the behavior of the models qualitatively. We consider $\alpha_{\text {irr }}=0.00,0.01,0.10$, and 1.00; and $\alpha_{\text {evap }} L_{\text {PSR }}=0.00,0.04,0.20$, and $1.00 L_{\odot}$. Typical early evolutionary tracks are presented in Figure 1, while the evolution of $\dot{M}$ for these CBSs is shown in Figure 2.

As stated above, during mass transfer, CBSs switch from accretion (LMXBs) to detachment conditions. The number of RLOFs is strongly dependent on $\alpha_{\text {irr }}$. The non-irradiated model (upper panel of Figure 2) behaves as an LMXB for almost the entire time period of Figure 2. In contrast, irradiated models allow for its detection either as an LMXB or radio pulsar with a companion (see the other panels of Figure 2) in a detached state. Although the number of RLOFs is strongly dependent on $\alpha_{\text {irr }}$, the duty cycle for detecting an LMXB or a radio pulsar is nearly constant. In the cases of $\alpha_{\text {irr }}=0.10$ and 1.00 , the cycles of mass transfer and detachments are visible in Figure 2. For $\alpha_{\text {irr }}=0.01$ the average width of the cycle is $\approx 1$ Myr for the RLOF and $\approx 2$ Myr detached. 


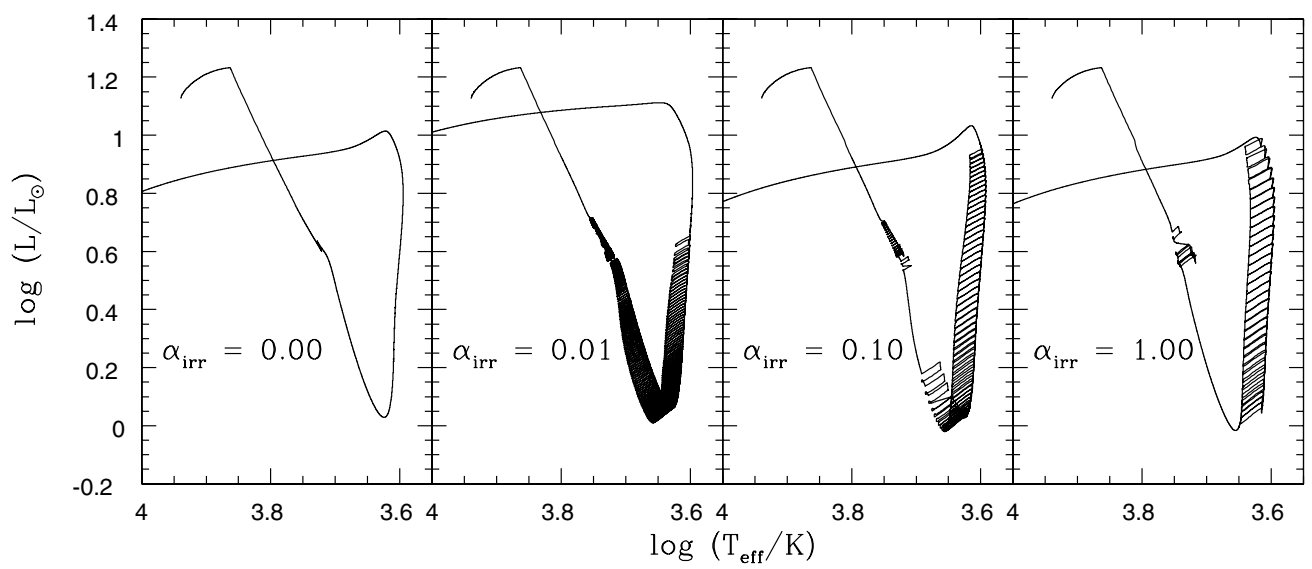

Figure 1. Early evolution of CBSs that initially have a normal, solar composition $M_{2}=2 M_{\odot}$ star and a "canonical" NS $\left(M_{1}=1.4 M_{\odot}\right)$ on a circular 1 day orbit. The effective temperature of the horizontal axis corresponds to the non-irradiated portion of the surface. The left panel shows the evolution of the system without irradiation feedback, while the other panels show cases with different strengths of irradiation, denoted by the value of $\alpha_{\text {irr }}$. The non-irradiated model suffers from one long RLOF. In contrast, irradiated models undergo a large number of RLOFs, separated by detached stages (See Figure 2). Evaporation is not important at the evolutionary stages depicted here.

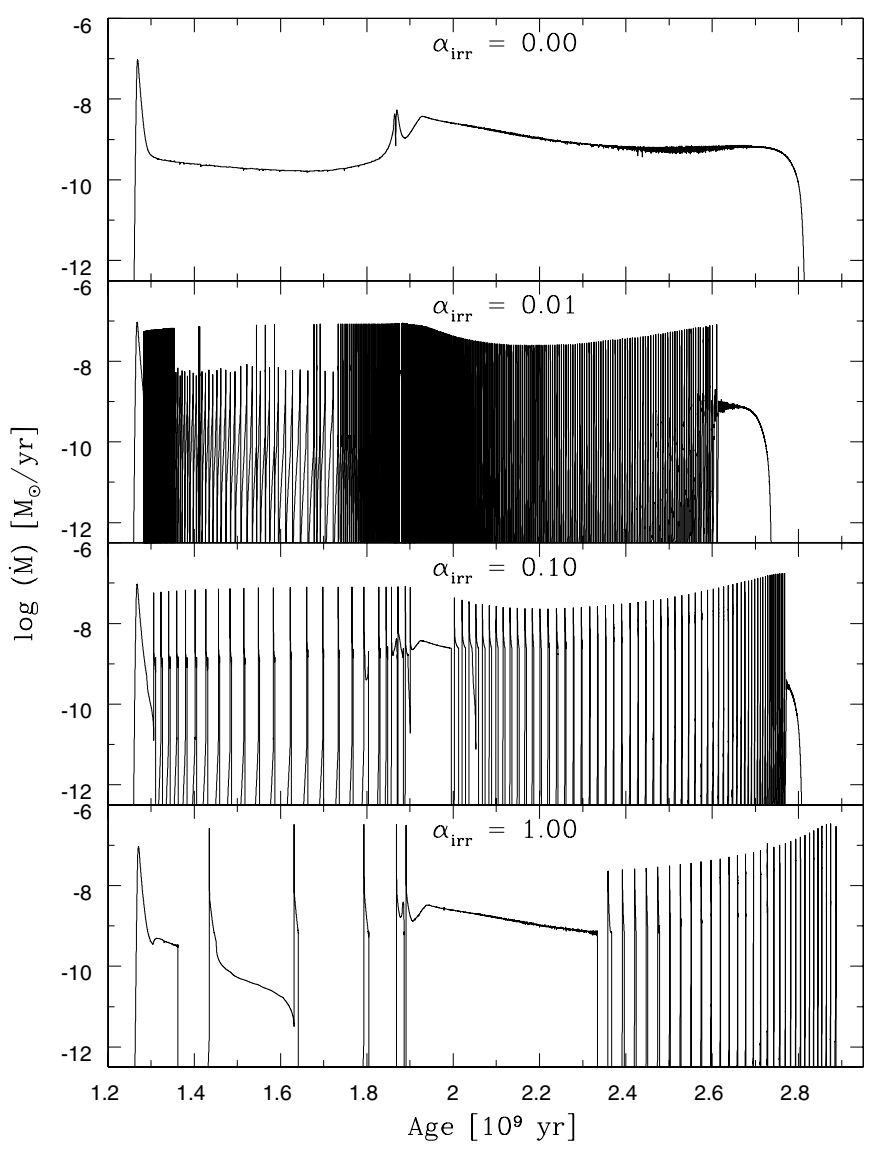

Figure 2. Evolution of the mass transfer rate for the CBSs included in Figure 1. The upper panel shows the case of the non-irradiated model, while the other panels correspond to the cases with different strengths of irradiation, denoted by the value of $\alpha_{\text {irr }}$. When the donor star exhausts its hydrogen core (at an age of $\approx 1.9 \mathrm{Gyr}$ ), mass transfer becomes momentarily stable. The NS accretion rate is limited by the Eddington rate $\left(\approx 2 \times 10^{-8} M_{\odot} \mathrm{yr}^{-1}\right)$.

In Figure 3, we present the evolution of CBSs together with available observed systems in the plane $M_{2}$ versus P. We restrict the data sample to those systems that contain one NS which has also undergone at least one RLOF. Those CBSs should have suffered strong tidal dissipation, and their orbits should be almost circular (Zahn 1977). The radio pulsar data were taken

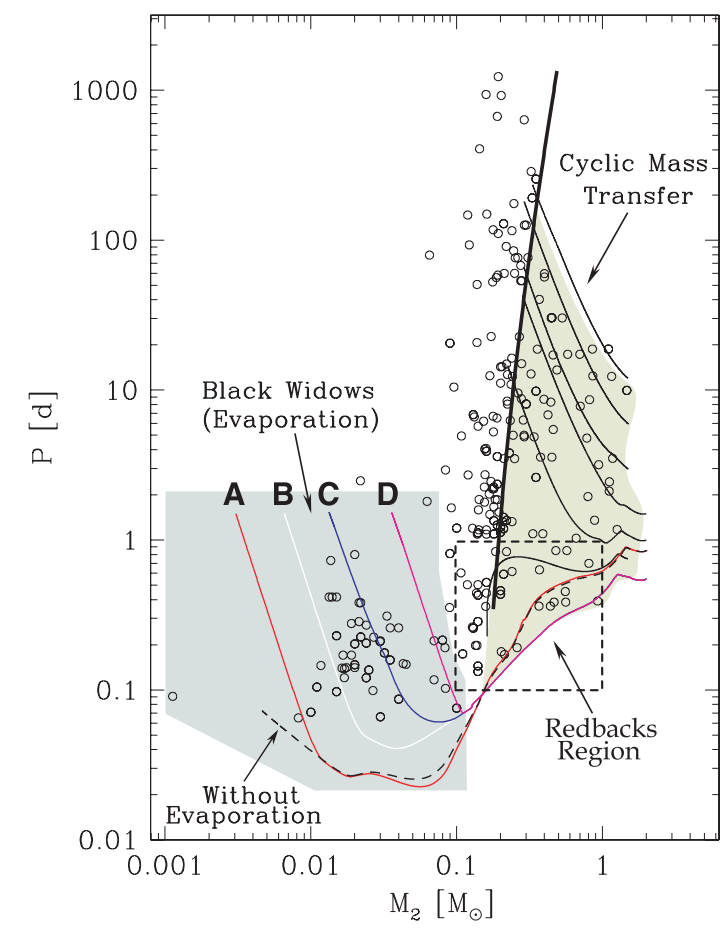

Figure 3. Donor mass vs. orbital period for CBSs, together with the corresponding observational data. Open dots denote minimum masses for radio pulsar companions. The $P\left(M_{2}\right)$ relationship is denoted by a thick solid line. On the right, thin solid lines denote the evolution of CBSs with initial donor masses and orbital periods of $1.50 M_{\odot}$ with $P_{i}=0.75,3.0,6.0$, and 12.0 days; and $2.00 M_{\odot}$ with $P_{i}=1.00$ and 1.50 days. All of them end close to the $P\left(M_{2}\right)$ curve. Tracks reaching the $\mathrm{BW}$ region (shaded on the left) correspond to an initial donor mass of $2.00 M_{\odot}$. Three of them have $P_{i}=0.55$ days and $\alpha_{\text {evap }} L_{\mathrm{PSR}}=0.04,0.20$, and $1.00 L_{\odot}\left(\mathrm{B}, \mathrm{C}\right.$, and $\mathrm{D}$, respectively) and another with $P_{i}=0.85$ days and $\alpha_{\text {evap }} L_{\mathrm{PSR}}=0.04 L_{\odot}(\mathrm{A})$. The shaded region on the right denotes conditions under which pulsed mass transfer occurs. The BW region is reached by systems that previously evolved through the redback region. Models without evaporation (see the long dashed line representing the case of $2.0 M_{\odot}$ with $P_{i}=0.85$ days) evolve to orbital periods that are too low, in conflict with observations.

(A color version of this figure is available in the online journal.)

from the ATNF database ${ }^{5}$ (Manchester et al. 2005), Freire's page $^{6}$ (although they correspond to other chemical abundances),

\footnotetext{
5 www.atnf.csiro.au/research/pulsar/psrcat

6 www.naic.edu/ p preire/GCpsr.html
} 


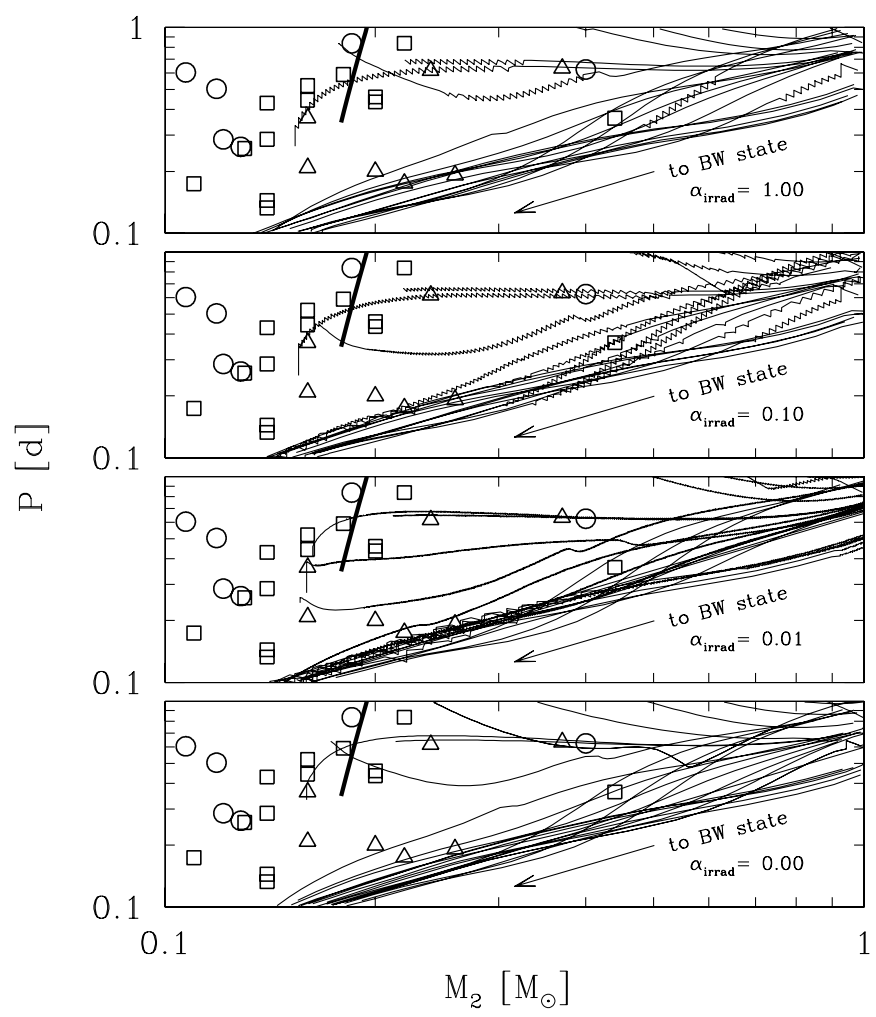

Figure 4. Region of Figure 3 where redbacks are located. Each panel corresponds to different irradiation conditions. Hollow circles, triangles, and squares correspond to observed systems taken from the ATNF database (Manchester et al. 2005), Roberts (2013), and Freire's home page, respectively. The thick solid line represents the low-mass end of the mass-period relation.

and Fermi gamma-ray sources (Cognard et al. 2011; Hessels et al. 2011; Ransom et al. 2011; Kerr et al. 2012; Ray et al. 2013; Breton et al. 2013). If for a given object more than one data sample is available, we plotted the larger value reported for the mass.

In Figure 3, CBSs with $P_{i} \gtrsim 1$ day evolve toward the $P\left(M_{2}\right)$ relation. If irradiation feedback is considered, there are stages at which the CBS is detached while the track passes through a region populated by several systems. CBSs that do not reach the $\mathrm{BW}$ region form a low-mass HeWD. We found that during the whole unstable mass transfer cycle the donor stars almost fill their Roche lobe. This is the result of the interplay between irradiation and orbital evolution. We call this state of donor stars quasi-RLOF hereafter. This represents an alternative evolutionary state for these companion objects. A very recently detected redback system, PSR J1723-2837 (Crawford et al. 2013), supports these results. Observations indicate that the Roche lobe of the donor star is almost completely filled, as predicted by these calculations. To be sure, there are other paths that arrive to the right of the $P\left(M_{2}\right)$ relation. These may actually be $\mathrm{CO}$ or HeCO WDs (see Tauris et al. 2000; Podsiadlowski et al. 2002). Our results indicate that there exists another, quite different, evolutionary path for these objects to occur.

In Figure 4, we show the redback region of Figure 3, where we include all computed models together with the observed systems. Redback systems with $P \gtrsim 6 \mathrm{hr}$ should form a lowmass HeWD-radio pulsar system. For shorter $P$, they are clearly $\mathrm{BW}$ progenitors. Many evolutionary tracks converge, evolving to the $\mathrm{BW}$ region marked in Figure 3. In short, to become a $\mathrm{BW}$ it is necessary to have characteristics similar to those that redbacks previously had. Nevertheless, not all redbacks evolve to BWs. Note that, in the redback region of Figure 4, irradiated models undergo cyclic mass transfer; so, we expect the detection of some binary systems to contain a radio pulsar. We find pulsed mass transfer at redback conditions for all considered initial donor masses and short $P$ values. Again, pulsed mass transfer is found especially for $\alpha_{\text {irr }}=0.01$, although it is also present for stronger irradiation regimes.

Another relevant item regarding redbacks and BWs is the mass of the underlying NS. A necessary condition for a CBS to become a redback is a short $P_{i}$. For such CBSs, mass transfer occurs at low rates. Thus, most of the matter is accreted by the NS, which becomes more massive. Although the mass growth of NSs depends on $\beta$, within the adopted assumptions we found redbacks with masses of $2.4 M_{\odot}$. This is consistent with recent reports (van Kerkwijk et al. 2011; Romani et al. 2012) claiming high NS masses for some BWs.

The BW region is reached by systems that evolved through the redback region (see Figure 3). Evolutionary tracks with and without irradiation feedback are hardly distinguishable from one another. However, this is not the case for evaporation. Models without evaporation evolve to have very low $P$, in conflict with observations. Evaporation makes the trajectories bend upward (in Figure 3), reaching the required range of $P$ values at ages shorter than the age of the universe. This is independent of the earlier operation of irradiation feedback, and should occur even if "radio ejection" prevents NS accretion. BW companions are semi-degenerate; thus, evaporation makes the star swell and the orbit widens fast enough to detach from its Roche Lobe (Benvenuto et al. 2012, 2013). The larger the $L_{\mathrm{PSR}}$, the steeper the trajectory. Interestingly, BW companions become fully convective for $\log \left(T_{\text {eff }} / \mathrm{K}\right) \lesssim 3.4$, independent of the evaporation rate.

\section{DISCUSSION AND CONCLUSIONS}

We computed the evolution of CBSs formed by an NS (behaving as a radio pulsar) and a normal donor star, for a range of donor masses for which we expect, as a final product, a low-mass HeWD or an ultra-compact system. We included $\mathrm{X}$-ray irradiation feedback and evaporation due to radio pulsar irradiation. These important ingredients allow for the occurrence of radio pulsars with companions in evolutionary stages in which the donor stars are undergoing episodic mass transfers and almost filling its Roche lobe (the quasi-RLOF state), stages that are widely different from a WD star. This seems to fit the case of J1723-2837 (Crawford et al. 2013), which otherwise remains hard to interpret.

We assumed that during RLOFs, the NS acts as an X-ray point source (Hameury \& Ritter 1997). This represents the simplest configuration, but not the only possible one (Ritter 2008). For example, the X-ray flux may come from an accretion disk. In any case, a sequence of RLOF and detachment cycles are expected. Note that for all the values of $\alpha_{\text {irr }}>0$ considered here we find a cyclic behavior for the mass transfer. Apparently, donor stars with convective envelopes are very sensitive to irradiation feedback, making the scenario presented here a robust one. This is the case provided that "radio ejection" is not operative; otherwise it would inhibit accretion onto the NS and irradiation feedback, too, though evaporation should occur.

Some of our models evolve to redback conditions. This is so for the whole range of initial masses and short initial orbital periods ( $P<1$ day). For all values of $\alpha_{\text {irr }}>0$ we find cyclic mass transfer episodes. A fraction of them form a low-mass 
HeWD, but others become BW progenitors. Therefore, our calculations indicate that all BWs should have passed through the redback region (although at such a stage they are not necessarily classified as redbacks if eclipses are not seen). Chen et al. (2013) studied this problem, based on models including evaporation. They found that only by fine tuning the evaporation rate for systems with $P<4 \mathrm{hr}$ will it be possible to find BWs that are redback descendants. This result is very different from the scenario described by our models.

As stated in Section 3, some BWs have been reported to harbor massive NSs. In our scenario where BWs are redback descendants, we infer that the NSs that behave as redbacks should also be massive. This is because most of the accretion onto NSs should have occurred prior to reaching redback conditions. We found CBSs corresponding to redbacks with NS masses as high as $2.4 M_{\odot}$.

In summary, we have calculated a set of evolutionary tracks showing the relevance of irradiation/evaporation stages for interacting CBSs. Although other relevant ingredients may still be lacking, we believe there is enough evidence that a unified picture of the redback/BW evolution is closely related to the consideration of these physical effects, allowing an interpretation of present observational evidence.

We deeply acknowledge our anonymous referee for a very constructive report that allowed us to markedly improve the original version of this Letter. O.G.B. thanks Leonardo Benvenuto for his assistance in the preparation of Figure 3. O.G.B. is a member of the Carrera de Investigador of the CIC-PBA Agency and M.A.D.V. is a member of the Carrera del Investigador Científico, CONICET, Argentina. J.E.H. has been supported by Fapesp (São Paulo, Brazil) and CNPq, Brazil funding agencies.

\section{REFERENCES}

Benvenuto, O. G., \& De Vito, M. A. 2003, MNRAS, 342, 50

Benvenuto, O. G., De Vito, M. A., \& Horvath, J. E. 2012, ApJL, 753, L33 Benvenuto, O. G., De Vito, M. A., \& Horvath, J. E. 2013, MNRAS, 433, L11

Breton, R. P., van Kerkwijk, M. H., Roberts, M. S. E., et al. 2013, ApJ, 769, 108

Büning, A., \& Ritter, H. 2006, A\&A, 445, 647

Büning, A., \& Ritter, H. 2004, A\&A, 423, 281

Burderi, L., Possenti, A., D’Antona, F., et al. 2001, ApJL, 560, L71

Chen, H.-L., Chen, X., Tauris, T. M., \& Han, Z. 2013, ApJ, 775, 27

Cognard, I., Guillemot, L., Johnson, T. J., et al. 2011, ApJ, 732, 47

Crawford, F., Lyne, A. G., Stairs, I. H., et al. 2013, ApJ, 776, 20

De Vito, M. A., \& Benvenuto, O. G. 2012, MNRAS, 421, 2206

Hameury, J.-M., \& Ritter, H. 1997, A\&AS, 123, 273

Hessels, J. W. T., Roberts, M. S. E., McLaughlin, M. A., et al. 2011, in AIP Conf. Proc. 1357, Radio Pulsars: An Astrophysical Key to Unlock the Secrets of the Universe, ed. M. Burgay et al. (Melvile, NY: AIP), 40

Kerr, M., Camilo, F., Johnson, T. J., et al. 2012, ApJL, 748, L2

Manchester, R. N., Hobbs, G. B., Teoh, A., \& Hobbs, M. 2005, AJ, 129, 1993

Podsiadlowski, P., Rappaport, S., \& Pfahl, E. D. 2002, ApJ, 565, 1107

Ransom, S. M., Ray, P. S., Camilo, F., et al. 2011, ApJL, 727, L16

Ray, P. S., Ransom, S. M., Cheung, C. C., et al. 2013, ApJL, 763, L13

Ritter, H. 2008, NewAR, 51, 869

Roberts, M. S. E. 2013, in IAU Symp. 291, Neutron Stars and Pulsars: Challenges and Opportunities after 80 years, ed. J. van Leeuwen (Cambridge: Cambridge Univ. Press), 127

Romani, R. W., Filippenko, A. V., Silverman, J. M., et al. 2012, ApJL, 760, L36

Romanova, M. M., Kulkarni, A. K., Long, M., \& Lovelace, R. V. E. 2008, in AIP Conf. Proc. 1068, A Decade of Accreting Millisecond X-ray Pulsars, ed. R. Wijnands et al. (Melvile, NY: AIP), 87

Ruderman, M., Shaham, J., \& Tavani, M. 1989, ApJ, 336, 507

Stevens, I. R., Rees, M. J., \& Podsiadlowski, P. 1992, MNRAS, 254, 19P

Tauris, T. M., \& Savonije, G. J. 1999, A\&A, 350, 928

Tauris, T. M., van den Heuvel, E. P. J., \& Savonije, G. J. 2000, ApJL, 530, L93

van Haaften, L. M., Nelemans, G., Voss, R., Wood, M. A., \& Kuijpers, J. 2012, A\&A, 537, A104

van Kerkwijk, M. H., Breton, R. P., \& Kulkarni, S. R. 2011, ApJ, 728, 95

Vaz, L. P. R., \& Nordlund, A. 1985, A\&A, 147, 281

Zahn, J.-P. 1977, A\&A, 57, 383 